\title{
Putative Equine Neorickettsiosis in a Mare from Southern Brazil
}

\author{
Victor Hugo Brunaldi Marutani, Selwyn Arlington Headley \& Ana Paula Frederico Rodrigues Loureiro Bracarense
}

\begin{abstract}
Background: This report describes the occurrence of equine neorickettsiosis (EN) in the northern region of Paraná, southern Brazil. EN is a non-contagious infectious disease caused by the Gram-negative bacterium, Neorickettsia risticii. Equine neorickettsiosis was previously known as Potomac horse fever and monocytic ehrlichiosis. The disease occurs predominantly in the USA and Canada; data relative to EN in Brazil is scarce. The aim of this study was to report the first case of putative EN in the state of Paraná due to a combination of IHC and molecular testing.

Case: A 2-year-old Quarter Horse was referred to a Veterinary Hospital with episodes of abdominal discomfort, fever, anorexia, tachycardia, and tachypnea. The animal reportedly demonstrated episodes of blackened and fetid diarrhea after the ingestion of hay. A treatment was established upon arrival at the veterinary hospital, but the mare died after 12 hours of monitoring. An autopsy examination performed soon after death revealed severe hyperemia of the mucosa of the cecum and colon, with multifocal cecal erosions and ulcerations. The principal histological lesion observed was necrotizing enterocolitis. Additional significant histopathologic lesions included widespread lymphoid depletion affecting the spleen, tonsils, and lymph nodes. An IHC assay designed to identify the antigens of $N$. helminthoeca (NH) in formalin fixed paraffin embedded (FFPE) tissues, identified antigens of intralesional neorickettsial organisms within macrophages of the mucosa of the colon. Additionally, a PCR assay designed to amplify the 16S rRNA gene of Neorickettsia, amplified the desired amplicon, but sequencing was frustrating.

Discussion: A putative diagnosis of equine neorickettsiosis was established due to the combination of epidemiological evidence, pathologic findings, immunohistochemical identification of intralesional antigens of neorickettsial agents, and amplification of the $16 \mathrm{~S}$ gene of Neorickettsia spp. by PCR. The gross and microscopic lesions described must be differentiated from lesions observed in equine salmonellosis and Clostridium difficile associated disease of horses. IHC was performed on FFPE intestinal tissue using an anti-NH hyperimmune serum as primary antibody due to high antigenic cross-reactivity between Neorickettsia spp. There was positive intracytoplasmic immunolabeling of macrophages in the mucosa of the large colon indicating the presence of neorickettsial antigens. The PCR amplified the desired amplicon from the colon fragment, but sequencing was frustrating due to poor quality product that resulted in an organism consistent with Methylobacterium spp., probably, a contaminant. EN show a seasonal and endemic tendency in USA, Uruguay and in southern Brazil, occurring in warmer months, as in the case reported. Evidence suggests that an ancient disease named "churrido" known for at least 100 years in southern Brazil and Uruguay is, in fact, equine neorickettsiosis and might have been introduced to North America from South America. Considering the epidemiological evidence and the worldwide distribution of NR, we suggest that EN is probably underdiagnosed in Brazil and that the etiology of equine enterocolitis should be more thoroughly investigated. Therefore, this report broadens the knowledge of EN in our region. In conclusion, considering the epidemiologic, pathologic, immunohistochemical and molecular evidence, and the specie-specificity of Neorickettsia spp., we report a putative case of EN in southern Brazil and the first report in Paraná state. IHC is a useful technique, less technically demanding and time consuming than bacterial culture and isolation, considered the gold standard; and can be used to diagnose EN when compatible pathologic evidence is present concomitantly.
\end{abstract}

Keywords: Potomac horse fever, Neorickettsia risticii, necrotizing enterocolitis, immunohistochemistry, PCR. 


\section{INTRODUCTION}

Equine neorickettsiosis (EN) is an infectious disease caused by the obligate intracellular bacterium Neorickettsia risticii (NR), family Anaplasmataceae $[6,7]$. This disease is also referred to as equine monocytic ehrlichiosis, Potomac horse fever, Ehrlichia colitis, and acute equine diarrhea syndrome $[15,18]$. EN was recognized as a distinct clinical entity in 1979 by veterinarians, in Maryland, USA, where it affected farm horses in regions close to the Potomac River [15]. Interestingly, EN was never diagnosed as a recognized disease prior to 1970 in USA and, it was hypothesized that EN might have been introduced to North America from South America [31], where NR has been identified as the causative agent of a disease locally known as "churrido equino" - a diarrheic disease of horses that probably occurred for at least 100 years in Uruguay and southern Brazil $[2,3,8]$.

Equine neorickettiosis is reported predominantly in the USA $[15,22,32]$ and Canada $[13,15,33]$. However, most of the previous reports available in database are based exclusively on serologic assays in horses without further evidence from either polymerase chain reaction (PCR)/sequencing or culturing the bacteria [31].

Available data concerning this disease is scarce in Brazil and up to the moment there are no studies related to immunohistochemical identification of $\mathrm{NH}$ in horses when public databases were consulted. Therefore, this study describes the first case of EN in the state of Paraná and provide the immunohistochemical characterization for the diagnosis of this disease.

\section{CASE}

A 2-year-old, female, Quarter horse was referred to a Veterinary Teaching Hospital in southern Brazil, during November 2016, after presenting severe signs of abdominal discomfort over the last two days. The animal reportedly had episodes of blackened and fetid diarrhea after the ingestion of hay. The mare was clinically examined, and samples were collected for laboratory tests (blood count, total plasma protein and fibrinogen dosage, hemogasometry, and peritoneal fluid analysis). A treatment was established upon arrival at the hospital and consisted of flunixin meglumine, gentamicin, penicillin potassium, lactated ringer solution with calcium and potassium chloride. Ten horses that consumed the same hay also had signs of abdominal discomfort, two of these died, but autopsy was performed only on the mare; the other diarrheic horses recovered spontaneously. These animals were maintained at a horse farm where horses from different geographical regions were housed.

The mare died $12 \mathrm{~h}$ after monitoring and an autopsy examination was performed soon after to determine the cause of death. Fragments of the large and small intestine, liver, lung, kidney, heart, mesenteric lymph nodes, mesenteric artery and vein, tonsils, esophagus, spleen and central nervous system were collected, immersed in $10 \%$ buffered formalin solution and routinely processed for histological evaluation (H.E.). FFPE tissue sections of the colon, mesenteric lymph nodes and spleen were selected for IHC. Additionally, fragments of the colon, mesenteric lymph nodes, and spleen were maintained frozen at $-20^{\circ} \mathrm{C}$ and used for molecular testing. A previously established protocol for the IHC identification of antigens of $\mathrm{NH}$ in FFPE tissues, using an anti-NH hyperimmune serum produced in immunized rabbits served as the primary antibody [11]. The IHC protocol was performed as previously described [20] using the anti-NH as primary antibody at 1:100 dilution. Positive controls consisted of an $\mathrm{NH}$ cellblock derived from pure cultures of this organism grown on DH82 cells and other paraffin embedded fragments known to be positive for NH [11]. The IHC assay was performed to detect antigens of $N$. helminthoeca due to elevated antigenic cross-reactivity with $N$. risticii [23]. The PCR assay was performed as described [12] using primers designed to amplify the 16S rRNA gene of Neorickettsia.

Clinically, the mare presented severe tachypnea, dyspnea and tachycardia; diarrhea, anorexia, excessive salivation and episodes of abdominal discomfort, terminating in lateral recumbency. The erythrogram revealed erythrocytosis $\left(10.2 \times 10^{6} / \mu \mathrm{L}\right.$, reference range: 5.5-9.5), while the leukogram demonstrated leukopenia $\left(1.4 \times 10^{3} / \mu \mathrm{L}\right.$, reference range: 6-12) with neutropenia $(280 / \mu \mathrm{L}$, reference range: $2.100-9.000)$ and monocytopenia $(84 / \mu \mathrm{L}$, reference range: $120-1.200)$. The biochemical assays revealed an increase in fibrinogen concentration $(600 \mathrm{mg} / \mathrm{dL}$, reference range: $<400$ ) without any alteration to the total plasma protein concentration. Hemogasometry revealed metabolic acidosis $\left(\mathrm{BE}_{(\text {ecf })}=-2.9 \mathrm{mEq} / \mathrm{L}\right.$, reference range: $0-6)\left(\mathrm{cHCO}_{3}=22.7 \mathrm{mEq} / \mathrm{L}\right.$, reference range: 24-30). No alteration was identified in the analysis of the peritoneal fluid. 
At autopsy, the animal had good nutritional status. All visible mucous membranes were congested and there was severe hydroperitoneum $(400 \mathrm{~mL})$. The main gross alterations were observed at the cecum and predominantly at the ventral colon. The serosa blood vessels of the colon were engorged, and the lumen was filled with fluid content of tan reddish color. The mucosa was diffusely hyperemic with areas of erosions and ulcerations ( 0.1 to $1.0 \mathrm{~cm}$ in diameter); furthermore, there was severe transmural edema and hemorrhage at the final region of the colon. The mucosa of the cecum was hyperemic with multifocal areas of erosions and ulcerations, ranging from 0.1 to $0.5 \mathrm{~cm}$ in diameter. Similar lesions were seen in the distal portion of the small intestine. Additionally, other significant alterations observed were moderate pulmonary edema and hemorrhage, moderate to severe multifocal to coalescing encephalic hemorrhage, and severe multifocal hemorrhagic renal infarcts.

The main microscopic findings were observed predominantly at the large and in some extent at small intestine and consisted of enterocolitis with diffuse and marked necrosis of the mucosa (Figure 1). The inflammatory cells were mixed but composed predominantly of macrophages. The intestinal villi were diffusely denuded with multifocal areas of fusion and intestinal crypts were frequently dilated. Intestinal vascular changes such as thrombosis and marked passive congestion and transmural interstitial edema were also observed in the large intestine. In addition, multifocal to coalescing areas of hemorrhage affecting the leptomeninges and the neuropil of the cerebellum, pituitary gland and brainstem were also observed.

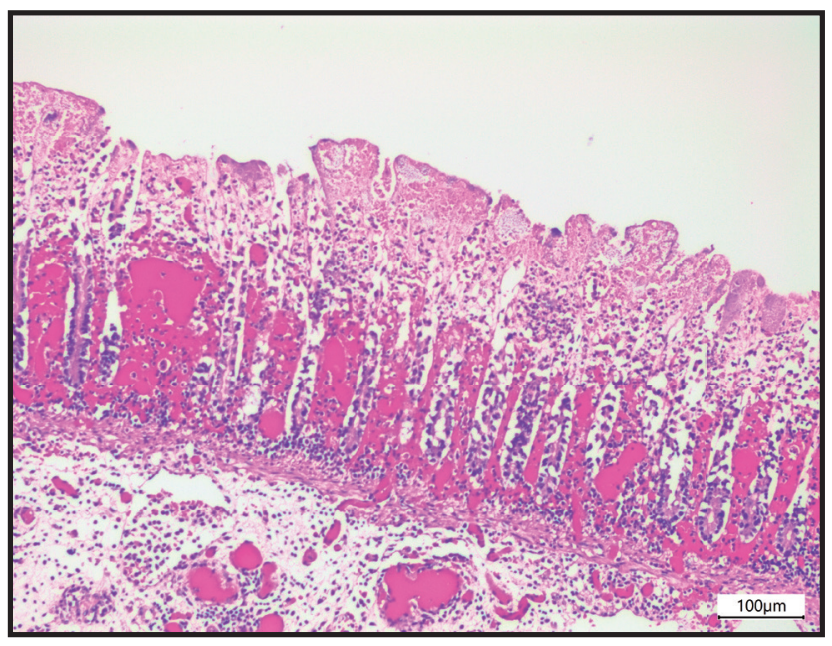

Figure 1. Intestinal tissue, horse. Severe and diffuse necrosis of enterocytes with villi atrophy and fusion; marked blood vessel congestion in mucosa and marked edema in submucosa [HE; 20x].
Other significant lesions included severe lymphoid depletion of the spleen, tonsils and lymph nodes.

By IHC, there was positive intracytoplasmic immunolabeling of intralesional macrophages to antigens of $N$. helminthoeca at the mucosa and lamina propria of the large intestine (Figure 2). The PCR assay designed to identify the $16 \mathrm{~S}$ gene of Neorickettsia amplified the desired amplicon from the colon that demonstrated positive immunoreactivity to antigens of NH. However, sequencing was frustrating due to poor quality DNA that resulted in an organism consistent with Methylobacterium spp.

\section{DISCUSSION}

A putative diagnosis of necrotizing enterocolitis associated with $N$. risticii in this mare was established due to a combination of pathologic findings, IHC identification of intralesional antigens of Neorickettsia, and amplification of the 16S gene of Neorickettsia spp. by PCR. The pathologic findings herein described can be attributed to EN but must be differentiated from the gross lesions observed in equine salmonellosis [14,29] and Clostridium difficile associated disease (CDAD) of horses [5,27].

Neorickettsial organisms are not easily identified in routine H.E. staining [30], however by Glemsa and toluidine blue staining it is possible to detect the neorickettsial microorganisms, mainly when cells are heavily infected [24]. Given this and the suggestive lesions of EN observed by histopathology, an IHC anti-NH assay $[10,11]$ was performed on FFPE intestinal tissue. We used an anti-NH hyperimmune serum as primary antibody due to antigenic cross-reactivity

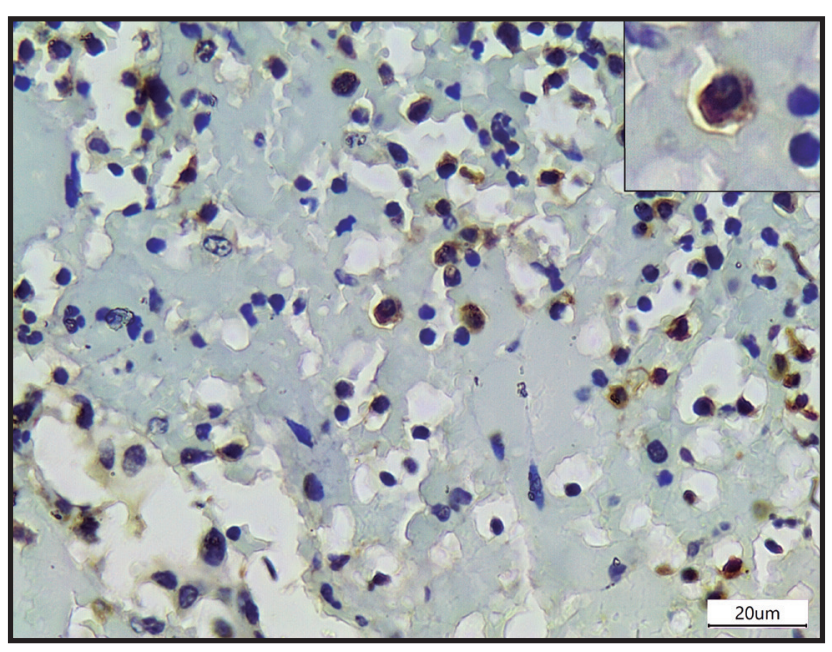

Figure 2. Intestinal tissue, horse. Positive intracytoplasmic immunolabelling anti-Neorickettsia antigens in macrophage at intestinal lamina propria [IHC; 100x]. 
between $N$. helminthoeca and $N$. risticii demonstrated by western blotting and immunofluorescence assays [23], and due to the similarity identified in phylogenetic analyses using several target genes of the Anaplasmataceae family [6,7], indicating that these organisms are closely related.

There was positive intracytoplasmic immunolabeling of macrophages located in the mucosa of the large colon indicating the presence of intralesional neorickettsial organisms. It is expected the immunolabeling in macrophages since $N$. risticii is an obligate intracellular bacteria that infects macrophages [4,24]. An in vitro study revealed that $N$. risticii binds to host macrophages through surface protein ligands, enters through a receptor-mediated endocytosis and viable or intact NR might prevent the phagosome-lysosome fusion, going on to multiply [16]. The internalization and proliferation of neorickettsial organisms depends on $\mathrm{Ca}^{2+}$-calmodulin-signaling [25] and protein tyrosine phosphorylation [34]. The spreading occurs by cell lysis and also through exocytosis by fusion of the inclusion membrane with the plasma membrane [7]. On the other hand, the intestinal villi were diffusely denuded due to severe intestinal damage with loss of viable enterocyte, and it might explain the absence of immunolabeling in enterocytes.

Additionally, the PCR assay was designed using a gene-specific primer for Neorickettsia spp. 16S rRNA [12] with the purpose to detect the presence of any neorickettsial organisms, rather than $N$. risticii specifically. The PCR amplified the desired amplicon from the colon fragment. Similar results occurred in other studies using PCR assay; in one of these neorickettsial DNA was amplified but the derived nucleotide sequences were short when compared with those deposited in GenBank [12]; however, cloning was used to certify the amplified product and other genes were used to corroborate the findings in that study. In another study, the quality of the PCR product was not adequate for sequencing but the diagnosis was associated with the IHC identification of intralesional Neorickettsia organisms [10]. In addition, the sequencing of the poor-quality DNA herein described resulted in an organism consistent with Methylobacterium spp; similar results were obtained in a study designed to identify $N$. helmintheoca by molecular biology [28]. Considering the obtained results herein described and a previous study [28], it must be highlighted that sequencing (to confirm the identification of the PCR product) and other methods (as histological analysis and IHC) must be performed, to establish the diagnosis of EN [28].

EN is seasonal and endemic in USA [1,9,19,21], Uruguay and in southern Brazil [2,8], occurring in warmer months. As in the case herein reported, the mare was affected during the summer (November). Interestingly it was suggested that a disease named "churrío" or "churrido", known for at least 100 years occurring during the summer in southern Brazil and Uruguay, is, in fact, equine neorickettiosis [8] and might have been introduced to North America from South America [31]. These findings associated with the serological evidence of the presence of N. risticii in other states from Brazil, such as Rio de Janeiro [26], Santa Catarina and São Paulo [17] and with the wide distribution of NR in North America, India and Europe [7,31]; suggest that EN is probably underdiagnosed in Brazil. In addition, the etiology of equine enterocolitis in Brazil should be more thoroughly investigated to associate the causative pathogens and reduce economic losses. Thus, this report broadens the knowledge of EN in our region.

In conclusion, considering the epidemiologic, pathologic, immunohistochemical and molecular evidences, and that natural infections of horses with $N$. helminthoeca or $N$. sennetsu have not been previously reported, we report a putative case of EN in southern Brazil and the first report in Paraná state. The lesions occurred mainly in the cecum and colon and were characterized by necrotizing enterocolitis. Lymphoid depletion at the spleen, tonsils and lymph nodes were also observed. Gastrointestinal disorders in horses are frequently observed and most of these are of infectious origin. EN is rarely reported in Brazil probably because it may be underdiagnosed and/or the etiology of equine enterocolitis is poorly investigated. Although the hyperimmune serum was designed to identify antigens of $\mathrm{NH}$, the IHC using anti-NH serum as primary antibody was able to identify Neorickettsia spp. antigens in horses with lesions suggestive of EN. Considering this evidence, a putative diagnosis of EN is herein described.

Acknowledgments. The authors are grateful to Dr. Thalita E.S. Oliveira and Nayara E. Viana for assisting with the immunohistochemical assay.

Declaration of interest. The authors declare that they have no conflict of interest. 


\section{REFERENCES}

1 Atwill E.R., Mohammed H.O., Dubovi E. \& Lopez J. 1992. Retrospective evaluation of factors associated with the risk of seropositivity to Ehrlichia risticii in horses in New York State. American Journal of Veterinary Research. 53(10): 1931-1934.

2 Coimbra H.S., Fernandes C.G., Soares M.P., Meireles M.C.A., Radamés R. \& Schuch L.F.D. 2006. Ehrlichiose monocítica eqüina no Rio Grande do Sul: aspectos clínicos, anátomo-patológicos e epidemiológicos-patológicos e epidemiológicos. Pesquisa Veterinária Brasileira. 26(2): 97-101.

3 Coimbra H.S., Schuch L.F.D., Veitenheimer-Mendes I.L. \& Meireles M.C.A. 2005. Neorickettsia (Ehrlichia) risticii no sul do Brasil: Heleobia spp. (mollusca: Hydrobilidae) e Parapleurolophocecous cercariae (trematoda: Digenea) como possíveis vetores. Arquivos do Instituto Biológico. 72(3): 325-329.

4 Cordes D.O., Perry B.D., Rikihisa Y. \& Chickering W.R. 1986. Enterocolitis caused by Ehrlichia sp. in the horse (Potomac horse fever). Veterinary Pathology. 23(4): 471-477.

5 Diab S.S., Rodriguez-Bertos A. \& Uzal F.A. 2013. Pathology and diagnostic criteria of Clostridium difficile enteric infection in horses. Veterinary Pathology. 50(6): 1028-1036.

6 Dumler J.S., Barbet A.F., Bekker C.P., Dasch G.A., Palmer G.H., Ray S.C., Rikihisa Y. \& Rurangirwa F.R. 2001. Reorganization of genera in the families Rickettsiaceae and Anaplasmataceae in the order Rickettsiales: unification of some species of Ehrlichia with Anaplasma, Cowdria with Ehrlichia and Ehrlichia with Neorickettsia, descriptions of six new species combinations and designation of Ehrlichia equi and 'HGE agent' as subjective synonyms of Ehrlichia phagocytophila. International Journal of Systematic and Evolutionary Microbiology. 51(6): 2145-2165.

7 Dumler J.S., Rikihisa Y. \& Dasch G.A. 2005. Family II Anaplasmataceae. In: Garrity G.M. (Ed). Bergey’s Manual of Systematic Bacteriology. vol. 2. 2nd edn. New York: Springer, pp.117-143.

8 Dutra F., Schuch L.F., Delucchi E., Curcio B.R., Coimbra H., Raffi M.B., Dellagostin O. \& Riet-Correa F. 2001. Equine monocytic Ehrlichiosis (Potomac horse fever) in horses in Uruguay and southern Brazil. Journal of Veterinary Diagnostic Investigation. 13(5): 433-437.

9 Goetz T.E., Holland C.J., Dawson J.E., Ristic M., Skibbe K., Keegan K.G., Johnson P.J., Schaeffer D.J. \& Baker G.J.1989. Monthly prevalence (in 1986) of antibody titers against equine monocytic ehrlichiosis in apparently healthy horses in Illinois. American Journal of Veterinary Research. 50(11): 1936-1939.

10 Headley S.A., de Oliveira T.E.S., Michelazzo M.M.Z., Fritzen J.T.T., Cubas Z.S., de Moraes W., Alfieri A.A. \& Vidotto O. 2018. Immunohistochemical and molecular evidence of putative Neorickettsia infection in coatis (Nasua nasua) from southern Brazil. Journal of Zoo and Wildlife Medicine. 49(3): 535-541.

11 Headley S.A., Kano F.S., Scorpio D.G., Tamekuni K., Barat N.C., Bracarense A.P.F.R.L., Vidotto O. \& Dumler J.S. 2009. Neorickettsia helminthoeca in Brazilian dogs: a cytopathological, histopathological and immunohistochemical study. Clinical Microbiology and Infection. 15(Suppl 2): 21-23.

12 Headley S.A., Scorpio D.G., Barat N.C., Vidotto O. \& Dumler J.S. 2006. Neorickettsia helminthoeca in dog, Brazil. Emerging Infectious Diseases. 12(8): 1303-1304.

13 Heller M.C., McClure J., Pusterla N., Pusterla J.B. \& Stahel S. 2004. Two cases of Neorickettsia (Ehrlichia) risticii infection in horses from Nova Scotia. The Canadian veterinary Journal. 45(5): 421-423.

14 Juffo G.D., Bassuino D.M., Gomes D.C., Wurster F., Pissetti C., Pavarini S.P. \& Driemeier D. 2017. Equine salmonellosis in southern Brazil. Tropical Animal Health and Production. 49(3): 475-482.

15 Knowles R.C., Anderson C.W., Shipley W.D., Whitlock R.H., Perry B. \& Davidson J.P. 1983. Acute equine diarrhea syndrome (AEDS): A preliminary report. Proceedings of the 29th Annual Convention - American Association of Equine Practitioners (Las Vegas, USA). pp.353-357.

16 Messick J.B. \& Rikihisa Y. 1993. Characterization of Ehrlichia risticii binding, internalization, and proliferation in host cells by flow cytometry. Infection and Immunity. 61(9): 3803-3810.

17 Moreira L.M.C., Cardoso K.M., Aboud-Dutra A.E., Ferrão C.M. \& Gazêta G.S. 2013. Frequency of Equine Monocytic Ehrlichiosis (EME) in Brazil. Pesquisa Veterinária Brasileira. 33(6): 697-699.

18 Mulville P. 1991. Equine monocytic ehrlichiosis (Potomac horse fever): a review. Equine Veterinary Journal. 23(6): 400-404.

19 Olchowy T.W., Ames T.R. \& Molitor T.W. 1990. Serodiagnosis of equine monocytic ehrlichiosis in selected groups of horses in Minnesota. Journal of the American Veterinary Medical Association. 196(12): 1967-1970. 
20 Oliveira T.E.S., Michelazzo M.M.Z., Fernandes T., de Oliveira A.G., Leme R.A., Alfieri A.F., Alfieri A.A. \& Headley S.A. 2017. Histopathological, immunohistochemical, and ultrastructural evidence of spontaneous Senecavirus A-induced lesions at the choroid plexus of newborn piglets. Scientific Reports. 7(1): 16555.

21 Palmer J.E., Whitlock R.H. \& Benson C.E. 1986. Equine ehrlichial colitis (Potomac horse fever): recognition of the disease in Pennsylvania, New Jersey, New York, Ohio, Idaho, and Connecticut. Journal of the American Veterinary Medical Association. 189(2): 197-199.

22 Pusterla N., Leutenegger C.M., Sigrist B., Chae J.S., Lutz H. \& Madigan J.E. 2000. Detection and quantitation of Ehrlichia risticii genomic DNA in infected horses and snails by real-time PCR. Veterinary Parasitology. 90(1-2): 129-135.

23 Rikihisa Y. 1991. Cross-reacting antigens between Neorickettsia helminthoeca and Ehrlichia species, shown by immunofluorescence and Western immunoblotting. Journal of Clinical Microbiology. 29(9): 2024-2029.

24 Rikihisa Y., Perry B.D. \& Cordes D.O. 1985. Ultrastructural study of ehrlichial organisms in the large colons of ponies infected with Potomac horse fever. Infection and Immunity. 49(3): 505-512.

25 Rikihisa Y., Zhang Y. \& Park J. 1995. Role of Ca2+ and calmodulin in ehrlichial infection in macrophages. Infection and Immunity. 63(6): 2310-2316.

26 Roier E.C.R., Costa R.L., Pires M.S., Vilela J.A.R., Santos T.M., Santos H.A., Baldani C.D. \& Massard C.L. 2016. Epidemiological survey of Neorickettsia risticii in equids from the State of Rio de Janeiro, Brazil. Pesquisa Veterinária Brasileira. 36(10): 939-946.

27 Songer J.G., Trinh H.T., Dial S.M., Brazier J.S. \& Glock R.D. 2009. Equine Colitis X associated with infection by Clostridium difficile NAP1/027. Journal of Veterinary Diagnostic Investigation. 21(3): 377-380.

28 Sykes J.E., Marks S.L., Mapes S., Schultz R.M., Pollard R.E., Tokarz D., Pesavento P.P., Lindsay L.L. \& Foley J.E. 2010. Salmon poisoning disease in dogs: 29 cases. Journal of Veterinary Internal Medicine. 24(3): 504-513.

29 Uzal F.A. \& Diab S.S. 2015. Gastritis, enteritis, and colitis in horses. Veterinary Clinics of North America: Equine Practice. 31(2): 337-358.

30 Uzal F.A., Plattner B.L. \& Hostetter J.M. 2016. Alimentary System. In: Maxie M.G. (Ed). Jubb, Kennedy, and Palmer's Pathology of Domestic Animals. 6th edn. St. Louis: Elsevier, pp.2-257.

31 Vaughan J.A., Tkach V.V. \& Greiman S.E. 2012. Neorickettsial endosymbionts of the digenea: diversity, transmission and distribution. Advances in Parasitology. 79: 253-297.

32 Wen B., Rikihisa Y., Fuerst P.A. \& Chaichanasiriwithaya W. 1995. Diversity of 16S rRNA genes of new Ehrlichia strains isolated from horses with clinical signs of Potomac horse fever. International Journal of Systematic and Evolutionary Microbiology. 45(2): 315-318.

33 Xiong Q., Bekebrede H., Sharma P., Arroyo L.G., Baird J.D. \& Rikihisa Y. 2016. An ecotype of Neorickettsia risticii causing Potomac horse fever in Canada. Applied and Environmental Microbiology. 82(19): 6030-6036.

34 Zhang Y. \& Rikihisa Y. 1997. Tyrosine phosphorylation is required for ehrlichial internalization and replication in P388D1 cells. Infection and Immunity. 65(7): 2959-2964.

http://seer.ufrgs.br/ActaScientiaeVeterinariae 